\section{Mortality of CAP reduced in the UK: is this enough?}

\author{
Antoni Torres, ${ }^{1}$ Carolina Garcia-Vidal $^{2}$
}

In 1930, pneumonia was the third most frequent cause of death in the USA. Now almost a century later, and despite the introduction of antibiotics in the 1950s, pneumonia remains the fourth most common cause of death worldwide. ${ }^{1}$ Community-acquired pneumonia (CAP) represents a major burden in terms of morbidity, mortality and health cost, as well as days of work lost.

In the last 10 years, guidelines for management of CAP have been published. ${ }^{2-4}$ One of the objectives of the guidelines is to improve outcomes (including mortality) when implemented. This was demonstrated, for example, by Dean et $a l^{5}$ showing a reduction in mortality from $14.2 \%$ to $11 \%$ in a series of hospitals in the USA after implementation of the 2001 American Thoracic Society CAP guidelines.

The British Thoracic Society (BTS) guidelines were published in $2009^{2}$ and they advised three key points for CAP quality standards: (1) to perform a chest X-ray within 4 hours of presentation, (2) to perform a mortality risk assessment using the CURB65 score and (3) to administer the first dosage of antibiotics within the first 4 hours after emergency department (ED) arrival. In parallel with the 2009 publication the BTS started a CAP audit programme. The audit was conducted from 2009 to 2012 and then subsequently in 2014. ${ }^{6}$ A total of 218 institutions submitted data for 24187 adults hospitalised for CAP. The main result of the study was a significant reduction of adjusted 30-day mortality (adjusted odds ratio: 0.86). The authors claim that this was due to a significant increase in the proportion of patients who received the first dosage within the first 4 hours of admission (57\%, 57\%, 60.8\%, $64.5 \%$ and $68.5 \%$ in $2009,2010,2011$, 2012 and 2013, respectively). This was also associated with a significant increase in the proportion of patients who had a

\footnotetext{
${ }^{1}$ Servei de Pneumologia, Hospital Clinic, Universitat de Barcelona, IDIBAPS, CIBERES, Barcelona, Spain; ${ }^{2}$ Servei de Malalties Infeccioses, Hospital Clinic, Barcelona, Spain

Correspondence to Dr Antoni Torres, Servei de Pneumologia, Villarroel 180, Barcelona 08036, Spain;
} atorres@clinic.ub.es chest X-ray within 4 hours of admission (76.5\%-80\% in 4 years). Other factors not reported by authors could have a played a role such as time to taken to present in ED after onset of symptoms, diagnosis and treatment of influenza and availability of critical care bed for invasive and non-invasive mechanical ventilation among others.

Other recent studies have found decreases in CAP mortality, although in a lower number of patients. In a recent investigation from a single centre in Spain, Simonetti et $a l^{7}$ observed a decrease in 30-day mortality (in the period 1995-1999) from $9.6 \%$ to $4.1 \%$ comparing 1995-1999 with 2010-2014. This decrease in mortality was observed despite patients being older and having a higher severity and more comorbidities in the second period.

A similar downward trend in mortality due to CAP has been reported in two previous studies ${ }^{8} 9$ using US national databases, where mortality due to CAP fell from $8.9 \%$ in 1993 to $4.1 \%$ in 2005 $(\mathrm{p}<0.001)$ in hospitalised patients ${ }^{8}$ and from $13.5 \%$ in 1987 to $9.7 \%$ in 2005 in a population of elderly inpatients and outpatients with $\mathrm{CAP}^{9}$

Two case-control studies showed reductions in mortality among patients with CAP. One study, ${ }^{10}$ which compared patients with CAP admitted to the intensive care unit in two periods (1995-2000 and 2005-2010), suggests that the decrease in mortality observed may be related to the follow-up of a sepsis management bundle derived from the Surviving Sepsis Campaign. In severe pneumococcal CAP, Gattarello et $a l^{11}$ showed a decreased mortality with time. Most importantly, they observed an increase from $25 \%$ to $87.5 \%$ of patients receiving the first dosage within the first 3 hours.

It seems that earlier administration of the first dosage of antibiotics is a beneficial and logical measure in reducing mortality. Older studies in $\mathrm{CAP}^{12}{ }^{13}$ confirm this statement and this is a quality measure for ED. ${ }^{14}$ However, we are not sure whether or not it is necessary for everybody with CAP. Menéndez et al ${ }^{15}$ and Yahav et $a l^{16}$ suggest that the beneficial effect on mortality when administering an early dosage should be focused on patients with severe sepsis and CAP, which accounts for $40 \%$ of admitted patients. We believe that in CAP we should use the sepsis view and consider the application of the sepsis six care bundle with all actions completed in 1 hour $\left(\mathrm{O}_{2}\right.$ administration, intravenous fluids, monitoring urine output, measuring lactate, taking blood cultures and giving appropriate antibiotic). In sepsis, there is clear evidence that mortality has decreased independently of the source. ${ }^{17}$

What we do not know from the BTS audit is whether the administration of the first dosage was done only when the chest X-ray was available. If this was the case, obtaining a chest X-ray is a potential barrier for early treatment in crowded EDs. We think that using severe sepsis scores ${ }^{18}$ or perhaps the recently published Quick SOFA score ${ }^{19}$ (very similar to CRB65) could be used without waiting for a chest-X-ray. In other words, we should find a way to detect or suspect those patients with severe sepsis due to CAP before performing a chest X-ray. The quick SOFA score needs to be validated in CAP.

One concern regarding the evaluation of the results of this study is the inclusion of patients coinciding in time with the $2009 \mathrm{H} 1 \mathrm{~N} 1$ pandemic and postpandemic season. Despite the authors discussing this limitation, it is important to note that some studies have reported an increased severity of influenza A (H1N1) 2009 pneumonia in the first postpandemic influenza season ${ }^{20}$ or even an estimate of respiratory and cardiovascular mortality associated with the 2009 pandemic influenza A H1N1 15 times higher than reported laboratory-confirmed deaths. ${ }^{21}$

Although 30-day mortality was reduced from $18.4 \%$ to $17.3 \%$ in the BTS audit study, mortalities observed are still very high and higher compared with other recent studies (not superior to $10 \%$ ) both in the overall population of $\mathrm{CAP}^{7}$ and in bacteremic pneumococcal pneumonia. ${ }^{22}$ Moreover, the rates of adherence to local antibiotic guidelines are low (55.6\% $57.3 \%)$ compared with previous studies. $^{23} 24$ The reasons for this high mortality and the low guideline adherences found in the BTS audit are unclear, but there is a good opportunity to study these findings and implement subsequent measures over the following years.

In summary, the results of the BTS audit are good news since they show a reduction in mortality probably due to a combined effect of both an increased implementation of chest-X-ray and first dosage of antibiotics before the first 
4 hours after ED arrival. Authors of the BTS audit have a great opportunity to further decrease mortality of hospitalised $\mathrm{CAP}$ in the ensuing years.

Competing interests None declared.

Provenance and peer review Commissioned; externally peer reviewed.

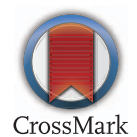

To cite Torres A, Garcia-Vidal C. Thorax 2016;71:979-980.

Published Online First 26 September 2016

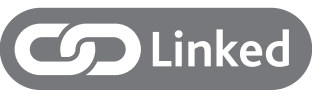

http://dx.doi.org/10.1136/thoraxjnl-2016-208937

Thorax 2016:71:979-980.

doi:10.1136/thoraxjnl-2016-209284

\section{REFERENCES}

1 GBD 2013 Mortality and Causes of Death Collaborators. Global, regional, and national age-sex specific all-cause and cause-specific mortality for 240 causes of death 1990-2013: a systematic analysis for the Global Burden of Disease study 2013. Lancet 2015;;385:117-71.

2 Mandell LA, Wunderink RG, Anzueto $A$, et al. Infectious Diseases Society of America/American Thoracic Society consensus guidelines on the management of community-acquired pneumonia in adults. Clin Infect Dis 2007;44(Suppl 2):S27-72.

3 Lim WS, Baudouin SV, George RC, et al. British Thoracic Society guidelines for the management of community acquired pneumonia in adults: update 2009. Thorax 2009;64 3):iii1-55.
4 Eccles S, Pincus C, Higgins B, et al, Guideline Development Group. Diagnosis and management of community and hospital acquired pneumonia in adults: summary of NICE guidance. BMJ 2014;349:g6722.

5 Dean NC, Silver MP, Bateman KA, et al. Decreased mortality after implementation of a treatment guideline for community-acquired pneumonia. Am J Med 2001;110:451-7.

6 Daniel P, Woodhead M, Welham S, et al. Mortality reduction in adult community acquired pneumonia in the UK (2009-2014): results from the British Thoracic Society audit programme. Thorax 2016:71:1061-3.

7 Simonetti AF, Garcia-Vidal C, Viasus D, et al. Declining mortality among hospitalized patients with community-acquired pneumonia. Clin Microbiol Infect 2016;22:567e1-7.

8 Ruhnke GW, Coca-Perraillon M, Kitch BT, et al. Trends in mortality and medical spending in patients hospitalized for community-acquired pneumonia: 1993-2005. Med Care 2010;48:1111-16.

9 Ruhnke GW, Coca-Perraillon M, Kitch BT, et al. Marked reduction in 30-day mortality among elderly patients with community-acquired pneumonia. Am J Med 2011;124:171-178.e1.

10 Georges H, Journaux C, Devos P, et al. Improvement in process of care and outcome in patients requiring intensive care unit admission for community acquired pneumonia. BMC Infect Dis 2013;13:196.

11 Gattarello S, Borgatta B, Solé-Violán J, et al. Decrease in mortality in severe community-acquired pneumococcal pneumonia: impact of improving antibiotic strategies (2000-2013). Chest 2014;146:22-31.

12 Meehan TP, Fine MJ, Krumholz HM, et al. Quality of care, process, and outcomes in elderly patients with pneumonia. JAMA 1997;278:2080-4.

13 Houck PM, Bratzler DW, Nsa W, et al. Timing of antibiotic administration and outcomes for Medicare patients hospitalized with community-acquired pneumonia. Arch Intern Med 2004;164:637-44.

14 Pines JM, Isserman JA, Hinfey PB. The measurement of time to first antibiotic dose for pneumonia in the emergency department: a white paper and position statement prepared for the American academy of Emergency Medicine. J Emerg Med 2009;37:335-40
15 Menéndez R, Torres A, Reyes $S$, et al. Initial management of pneumonia and sepsis: factors associated with improved outcome. Eur Respir J 2012;39:156-62.

16 Yahav D, Leibovici L, Golberg E, et al. Time to first antibiotic dose for patients hospitalised with community-acquired pneumonia. Inter I Antimicrob Agents 2013;41:410-13.

17 Shankar-Hari M, Harrison DA, Rowan KM. Differences in Impact of Definitional Elements on Mortality Precludes International Comparisons of Sepsis Epidemiology-A Cohort Study Illustrating the Need for Standardized Reporting. Crit Care Med Published Online First: 27 Jan 2016. doi:10.1097/ CCM.0000000000001876

18 Dellinger RP, Levy MM, Rhodes A, et al. Surviving Sepsis Campaign: international guidelines for management of severe sepsis and septic shock, 2012. Intensive Care Med 2013;39:165-228.

19 Shankar-Hari M, Phillips GS, Levy ML, et al. Developing a new definition and assessing new clinical criteria for septic shock: for the third international consensus definitions for sepsis and septic shock (Sepsis-3). JAMA 2016;315:775-87.

20 Viasus D, Cordero E, Rodríguez-Baño J, et al. Changes in epidemiology, clinical features and severity of influenza A (H1N1) 2009 pneumonia in the first post-pandemic influenza season. Clin Microb Infect 2012;18:E55-62.

21 Dawood FS, Iuliano AD, Reed C, et al. Estimated global mortality associated with the first 12 months of 2009 pandemic influenza A H1N1 virus circulation: a modeling study. Lancet Infect Dis 2012;12:687-95.

22 Amaro R, Liapikou A, Cilloniz C, et al. Predictive and prognosic factors in patients with blood-culture-positive community-acquired pneumonia. Eur Respir J 2016;48:797-807.

23 Dambrava PG, Torres $A$, Vallès $X$, et al. Adherence to guidelines' empirical antibiotic recommendations and community-acquired pneumonia outcome. Eur Respir J 2008;32:892-901.

24 Menéndez R, Torres A, Zalacaín R, et al. Guidelines for the treatment of community-acquired pneumonia. Predictors of adherence and outcome. Am J Respir Crit Care Med 2005;172:757-62. 